\title{
Perceived Egocentric Distances in Real, Image-Based, and Traditional Virtual Environments
}

\author{
Peter Willemsen Amy A. Gooch \\ School of Computing, University of Utah \\ URL: www.cs . utah.edu/immersive/perception.html \\ E-mail: $\{$ willemsn | gooch\}@cs.utah.edu
}

\begin{abstract}
In virtual environments, perceived egocentric distances are consistently underestimated when compared to the same distance judgments in the real world. The research presented in this paper explores two possible causes for the compressed distance perception in virtual environments: (1) real-time computer graphics rendering, and (2) immersive display technology. Our results show there is a significant difference in distance judgments between real and virtual environments. However, the differences between distance judgments in virtual photographic panorama environments and traditionally rendered virtual environments are small, suggesting that the display device is affecting distance judgments in virtual environments.
\end{abstract}

\section{Introduction}

The perceived distance to objects in virtual environments when viewed through head-mounted display (HMD) systems have been shown to be consistently underestimated. The same distance judgments conducted in the physical world have been shown to be quite accurate for distances up to about 25 meters [3]. The exact reason for this disparity and compression of space in a virtual environment is unknown. In this paper, we report results from experiments designed to explore the cause of this perceived distance compression. There are two likely causes for the perceived compression of space in virtual environments. Either information is missing from the scene's rendering, or the underestimation of distance stems from the display system.

\section{Experiment Description}

We probed subjects' perception of absolute egocentric distance [2] using a directed action task [4, 5, 6, 7] in which subjects walked without vision to a previously viewed target. The target was placed on the ground at distances of $2 \mathrm{~m}, 3.5 \mathrm{~m}$, and $5 \mathrm{~m}$. All subjects were college age, were given a stereo-gram eye test, and had normal or corrected to normal vision with contacts. Our subject pool consisted of 6 females and 6 males. Each subject performed a total of 36 trials in three conditions: (1) real hallway, (2) vir- tual 360 degree stereo, photographic panorama of the real hallway displayed at the highest available resolution in our HMD, and (3) fully polygonal, computer graphics rendering of the same hallway displayed in our HMD. All environmental conditions were presented binocularly. Three training trials were conducted for each condition followed by three trials at each of the three distances. No feedback was provided during any phase of the experiment. The order in which the environment conditions were presented was randomized over the subject pool. Target distance ordering was also randomized.

Prior to each experimental condition, subjects practiced blind walking. Training consisted of the subjects walking blindfolded in a hallway for approximately 5 minutes. For each trial, subjects were shown a target located on the floor. The target used in all of these experiments was a bright orange frisbee. Subjects were instructed to obtain a good image of the target and their local surroundings. Each subject was told that a "good image" is obtained if when the subject closed their eyes, he or she would still be able to "see" the environment, and most importantly, the target. Cognitive maps such as these have been extensively explored in the perception literature, as well as some virtual environment studies [1]. Subjects were allowed to rotate their head about their neck, but were instructed not to move their head from side to side or back and forth, in order to reduce depth cues resulting from motion parallax. Once a good image was achieved, subjects either blindfolded themselves or the HMD screen was cleared. Subjects were directed to then start walking toward the target in a purposeful and decisive manner and stop walking when they felt their feet were where the target had been observed.

\subsection{Equipment and Software}

The equipment used in this experiment consisted of an nVision Datavisor HiRes HMD with interlaced 1280x1024 resolution, full color, stereo, and a 52 degree diagonal field of view. Both computer generated environments were rendered on an SGI Onyx2 R12000 with two IR2 rendering pipelines. A fixed interpupil distance of $6 \mathrm{~cm}$ was used for the geometric and panoramic virtual environments. We 


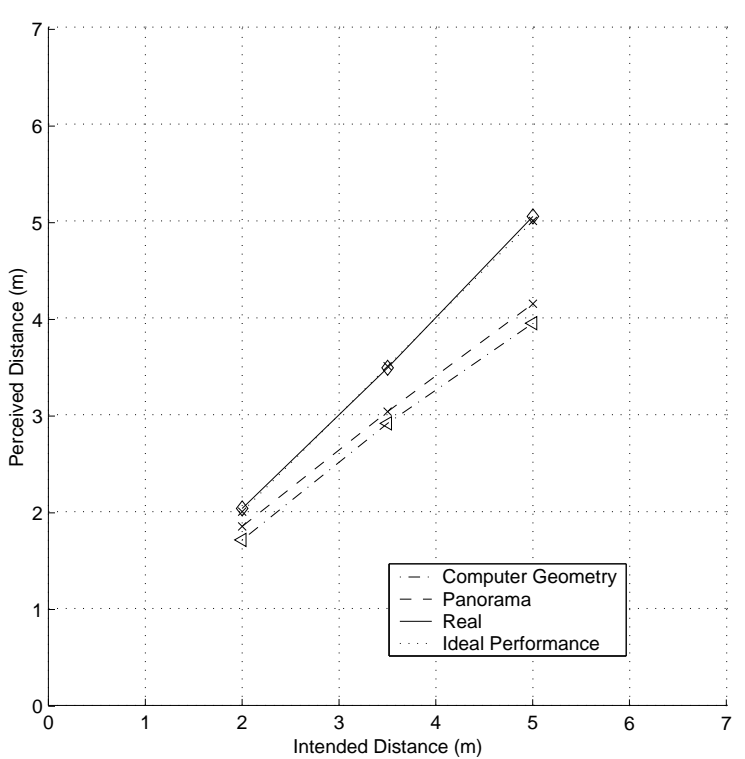

Figure 1. Comparison of results.

recorded the interpupil distance for each subject with the average interpupil distance being $6.22 \mathrm{~cm}$. Due to the lack of precise control over image position and accommodation in HMDs and the adjustments users can make which cannot be determined by polling the HMD, we can only roughly control the interpupil distance of the HMD. The geometrically modeled virtual environment ran at no less than 22 frames per second. The polygon count in this model was approximately 28,000 polygons utilizing 35 texture files. The panoramic environment was created from stereo photographs taken at a range of eye heights spaced at $5 \mathrm{~cm}$ intervals with a camera body equipped with a $12 \mathrm{~mm}$ lens providing 112 degrees vertical by 90 degrees horizontal field of view in portrait mode. The panorama environment ran at no less than 40 frames per second.

\section{Results and Conclusions}

We analyzed the data using a 3(environment) x 3(distance) repeated measures ANOVA. The results from our experiments indicate that there is a significant difference $\left(p_{\text {pano }}=0.004\right.$ and $\left.p_{c g}=0.001\right)$ in performance between the real world condition and both of the virtual environment conditions. The plot in Figure 1 provides a direct comparison of the data from the three environments.

The distance judgments based on actual images (i.e. panoramic environment) were slightly better than those based on the computer geometry, but the differences were small and not statistically significant $(p=0.392)$. This leads us to believe that the quality of graphics does not matter as much as previously thought and that the display is playing a large roll in the compression of perceived distance. While we did confirm results obtained by previous research that perception in virtual environments is foreshortened compared to perception in real environments, it is worth noting that the distance judgments made in the computer geometry environment of our study were more accurate than those reported by most other researchers $[3,8,9]$. It was expected, prior to the experiment, that the average perceived depth in the computer geometry condition would have been less accurate, thus resulting in statistical significance between the image-based panorama environment and the computer geometry condition. This increased sense of space may be due, in part, to the geometric complexity found in our model of the hallway, in comparison with simpler geometries used in other research. Additionally, the high resolution of our display system may have also contributed to the more accurate perception.

Our paper is the first to evaluate egocentric distance perception using high-resolution photographs in an immersive display. Our results provide us with confidence that our experimental methodology is correct since the real hallway data are consistent with what has been found by both the spatial perception and computer graphics research communities. Because the difference in perception between the computer geometry and photographic panorama conditions was not statistically significant, it suggests that the headmounted display is one source of the compression in virtual environments. While these conclusions are interesting, understanding the causes and magnitude of spatial compression in virtual environments requires substantial additional investigation and should be explored thoroughly.

\section{Acknowledgments}

Thanks to Alias|Wavefront for their generous donation of Maya Complete, which we use to create the geometric models for our experiments. This material is based upon work supported by the National Science Foundation under Grants CDA9623614 and IIS-0080999. Any opinions, findings, and conclusions or recommendations expressed in this material are those of the author(s) and do not necessarily reflect the views of the sponsors.

\section{References}

[1] M. Billinghurst and S. Weghorst. The use of sketch maps to measure cognitive maps of virtual environments. Virtual Reality Annual International Symposium, pages 40-47, 1995.

[2] J. E. Cutting and P. M. Vishton. Perceiving layout and knowing distance: The integration, relative potency and contextual use of different information about depth. In W. Epstein and S. Rogers, editors, Perceptual of Space and Motion, pages 69-117. Academic Press, New York, 1995.

[3] J. M. Knapp. The visual perception of egocentric distance in virtual environments. PhD thesis, University of California Santa Barbara, Dec. 1999.

[4] J. M. Loomis, J. A. Da Silva, N. Fujita, and S. S. Fukusima. Visual space perception and visually directed action. Journal of Experimental Psychology: Human Perception and Performance, 18:906-921, 1992.

[5] J. W. Philbeck and J. M. Loomis. Comparison of two indicators of perceived egocentric distance under full-cue and reduced-cue conditions. Journal of Experimental Psychology: Human Perception and Performance, 23(1):72-85, 1997.

[6] J. W. Philbeck, J. M. Loomis, and A. C. Beall. Visually perceived location is an invariant in the control of action. Perception and Psychophysics, 59(4):601-612, 1997.

[7] J. J. Rieser, D. H. Ashmead, C. R. Taylor, and G. A. Youngquist. Visual perception and the guidance of locomotion without vision to previously seen targets. Perception, 19:675-689, 1990.

[8] B. G. Witmer and P. B. Kline. Judging perceived and traversed distance in virtual environments. Presence: Teleoperators and Virtual Environments, 7(2):144 167, April 1998.

[9] B. G. Witmer and W. J. Sadowski, Jr. Nonvisually guided locomotion to a previously viewed target in real and virtual environments. Human Factors, 40:478488, 1998. 